\title{
Recent Innovations on Drawing of Random Multi-digit Numbers: Working Principles
}

\author{
Dhritikesh Chakrabarty* \\ Department of Statistics, Handique Girls' College, Gauhati University, India
}

Submission: May 07, 2018; Published: September 04, 2018

*Corresponding author: Dhritikesh Chakrabarty, Department of Statistics, Handique Girls' College, Gauhati University, Guwahati-781001, Assam, India, Email: dhritikeshchakrabarty@gmail.com

\section{Abstract}

Recently, a lot of studies have been done on

(i) Examination of randomness of existing tables of random numbers,

(ii) The construction of tables of random two-digit numbers \& of random three-digit numbers and

(iii) Searching for methods of drawing of m-digit random numbers ( $m=4,5,6,7,8,9)$ from table(s) of random two-digit numbers and/or from table(s) of random three-digit numbers and/or from suitable combination(s) of table(s) of random two-digit numbers and table(s) of random threedigit numbers from a table of random two-digit numbers and/or from a table of random three-digit numbers and/or from a combination of the two which one is suitable.

Keywords: Table of random two-digit numbers; Table of random three-digit numbers; Random multi-digit numbers; Working principles, Method of drawing

\section{Introduction}

The major contributions in the construction of tables of random numbers have been found to be made by Tippett [1], Fisher \& Yates [2], Hald [3], Hill \& Hill [4], Kendall \& Smith [5,6], Mahalanobis [7], Manfred [8], Moses \& Oakford [9], Quenouille [10], RAND Corporation [11], Rao, Mitra \& Matthai [12], Rohlf \& Sokal [13], Royo \& Ferrer [14], Snedecor and Cochran [15] and others who constructed tables of random numbers of different types. Among these tables, the four tables due to Tippett, Kendall \& Smith, Fisher \& Yates and RAND Corporation are found to be widely used in drawing of simple random sample (with or without replacement) from a population. The tables of random numbers that had been constructed are of two-digit numbers, three-digit numbers and four-digit numbers only. No table of random $\mathrm{m}$-digit numbers is available till now for $\mathrm{m}>5$.

In the mean time, some studies have been done on testing of the degree of randomness of tables of random numbers due to Tippett, Fisher \& Yates, Kendall \& Smith and RAND Corporation [16-29]. The degree of randomness of the tables has been examined by some standard statistical testing methods as well as on the philosophy behind the theoretical definition of probability due to Chakrabarty [30-39]. From the studies, degree of randomness of the tables has been found not so satisfactory. Due to this, research has been done on the construction of random two-digit numbers and of random three-digit numbers
[40-47]. However, no table of random $\mathrm{m}$-digit numbers is available till now for $m>5$. But, there is or there may be necessity of drawing of random five-digit numbers, random four-digit numbers, random seven-digit numbers etc.

Due to difficulties in the construction of tables of these types of random numbers, techniques have been innovated for drawing of random m-digit numbers $(\mathrm{m}=4,5,6,7,8,9)$ from table(s) of random two-digit numbers and/or from table(s) of random three-digit numbers and/or from suitable combination(s) of table(s) of random two-digit numbers \& table(s) of random three-digit numbers [48-57]. Working principles of drawing of $\mathrm{m}$-digit random numbers $(\mathrm{m}=4,5,6,7,8,9)$, from a single table of random two-digit numbers and/or from a single table of random three-digit numbers and/or from a combination of the two which one is suitable, have been extracted from the findings of these studies. This paper describes a brief presentation of these principles.

\section{Method of Drawing of Random Numbers}

The methods of drawing of random numbers are discussed here in the context of the two tables of random numbers, one of random two-digit numbers and the other of random three-digit numbers constructed by Chakrabarty. The two tables contain 10000 random occurrences of the 100 two-digit numbers 
$00,01,02,03$ 98, 99 and 10000 random

occurrences of three-digit numbers

$000,001,002,003$ 998, 999 respectively.

\section{Drawing of Random Two-digit Numbers}

Let us consider a table of random two-digit numbers. If $n$ random two-digit numbers are drawn from the table then

the drawn numbers may be distinct and

the drawn numbers may not be distinct.

Drawing of random two-digit numbers consists of the two basic tasks namely

selection of the starting position at random and selection of the direction (right or left) of movement at random $[45,58]$.

Drawing of distinct random two-digit numbers

In order to obtain the $\mathrm{n}$ random two-digit numbers, from a table of random two-digit numbers, one is to proceed with the following steps $[45,58]$.

Select the position, from where one will have to start the drawing of the numbers, at random. Since the table contains 10000 random occurrences of the 100 two-digit numbers, there are 10000 positions of the numbers namely $(0000,0001,0002$, 9999).

In selecting the starting position, one thus can apply some usual manual randomization technique of drawing one number from among the numbers $(0000,0001,0002$, 9999).

\section{One method of drawing of such number is as follows:}

Take a set of 10 identical small balls distinguishing them by marking with the 10 digits

$0,1,2,3,4,5,6,7,8,9$ and put them inside a opaque container, say $C_{1}$.

Similarly, take another set of 4 identical small distinguishing them by marking $L, R, M_{1}, M_{2}$ respectively and another opaque container, say $C_{2}$. Now, draw one ball at random from the container $C_{1}$ containing the 10 balls and note down digit appeared on it. Let the digit be $d_{1}$.

Next, draw another ball at random from the container $C_{1}$ containing the same 10 balls and note down digit appeared on it. Let the digit be .

Then, draw one ball at random from the container $C_{2}$ putting 2 balls marked with L \& R inside it.

If the drawn ball is $\mathrm{R}$ then put the digit at the right position of and if the drawn ball is $\mathrm{L}$ then put the digit at the left position of
Thus if the ball $\mathrm{R}$ appears then the selected two-digit number will be $d_{1} d_{2}$ and if the ball $L$ appears then the selected twodigit number will be . Let the selected two-digit number be .

Next, draw another ball at random from the container $C_{1}$ containing all the 10 balls and note down digit appeared on it. Let the digit be .

Then, draw one ball at random from the container $C_{2}$ putting 3 balls marked with $L, M_{1} \& R$ inside it and put the digit at the left position of if the drawn ball is $L$, the middle position of if the drawn ball is $M_{1} \&$ the right position of if the drawn ball is $R$. Thus the selected three-digit number will be $d_{3} d_{2} d_{1}$ or $d_{2} d_{3} d_{1}$ or $d_{2} d_{1} d_{3}$ in accordance with the selected ball is $L$ or $M_{1}$ or $R$.

Let the selected three-digit number be .

Finally, draw another ball at random from the container $C_{1}$ containing all the 10 balls and note down digit appeared on it. Let the digit be

Then, draw one ball at random from the container $C_{2}$ putting 4 balls marked with $L, M_{1}, M_{2} \& R$ inside it and put the digit at the left position of if the drawn ball is $L$, the $1^{\text {st }}$ middle position (from left) of if the drawn ball is $M_{1}$, the $2^{\text {nd }}$ middle position (from left) of if the drawn ball is $M_{2}$ \& the right position of if the drawn ball is $\mathrm{R}$.

Thus the selected four-digit number will be or or or in accordance with the selected ball is $\mathrm{L}$ or $\mathrm{M}_{1}$ or $\mathrm{M}_{2}$ or $R$.

This selected number will be the required starting position.

Let the ith position be selected as the starting position. Draw the number that occurs at the ith position in the table.

Chose whether to move towards left or towards right. The choice can be made at random by performing a random binary trial (for example, by tossing of an unbiased coin or by drawing a number from the container $C_{2}$ putting two identical balls, marked with $\mathrm{L}$ and $\mathrm{R}$ respectively, inside it.

If it is chosen to move towards right, draw the numbers occurred at the positions

$$
i, i+1, i+2, \ldots \ldots \ldots \ldots, i+n-1
$$

in the table to obtain the $\mathrm{n}$ random two-digit numbers.

If it is chosen to move towards left, draw the numbers occurred at the positions

$$
i, i-1, i-2, \ldots \ldots \ldots \ldots, i-n+1
$$

in the table to obtain the $\mathrm{n}$ random two-digit numbers.

It may occur that some number or numbers among those drawn may be occurred twice. In that situation, retain only one occurrence of them and draw additional numbers appeared at the consecutive positions in the table as per requirement. If $\mathrm{k}$ additional numbers are required to draw, then draw the numbers occurred at the positions 


$$
i+n, i+n+1, i+n+2, \ldots \ldots \ldots ., i+n+k-1
$$

if it is chosen to move towards right and draw the numbers occurred at the positions

if it is chosen to move towards left.

$$
i-n, i-n-1, i-n-2, \ldots \ldots \ldots . ., i-n-k+1
$$

Drawing of random two-digit numbers (not necessarily distinct)

In order to draw $\mathrm{n}$ random two-digit numbers which need not necessarily be distinct, from a table of random two-digit numbers, one is to proceed with the following steps $[45,56]$ :

Select one position from where to start at random by the similar method as in the case of drawing of distinct random twodigit numbers mentioned above. Let the ith position be selected.

Draw the number that occurs at the ith position in the table.

Chose the length of jump that is to be 101 or more and 199 or less at random. It can be chosen by some usual manual randomization technique of drawing one number from among the numbers

$101,102,103$ 198, 199.

Let the selected length of jump be 1 .

The random selection of the length of the jump can be done by similar method as done in the selection of the starting position.

Choose whether to jump towards left or towards right. The choice can be made by the same method as in the earlier case.

If it is chosen to jump towards right, draw the numbers occurred at the positions

$$
i, i+l, i+2 l, \ldots \ldots \ldots \ldots . . ., i+(n-1) l
$$

in the table to obtain the required $\mathrm{n}$ random two-digit numbers.

If it is chosen to move towards left, draw the numbers occurred at the positions

$$
i, i-l, i-2 l, \ldots \ldots \ldots \ldots, i-(n-1) l
$$

in the table to obtain the required $\mathrm{n}$ random two-digit numbers.

Drawing of random three-digit numbers

Let us consider a table of random three-digit numbers. If $n$ random three-digit numbers are drawn from the table then the drawn numbers may be distinct or may not be distinct.

Drawing of random three-digit numbers consists of the two basic tasks namely

selection of the starting position at random \& selection of the direction (right or left) of movement at random $[46,56]$.

Drawing of distinct random three-digit Numbers

In order to obtain the $\mathrm{n}$ distinct random three-digit numbers, from a table of random three-digit numbers, one is to proceed with the following steps $[46,56]$ :

Select the position, from where to start, at random. Since the table contains 10000 random occurrences of the 1000 threedigit numbers, accordingly there are 10000 positions of the numbers namely $(0000,0001,0002, \ldots \ldots \ldots \ldots \ldots . . .9998,9999)$. The starting position can be selected by the same method as in the case of drawing of random two-digit numbers.

Let the ith position be selected in the earlier step. Draw the number that occurs at the ith position in the table.

Chose whether to move towards left or towards right. The choice can be made at random by a binary trial e.g. by tossing of an unbiased coin or by drawing a number from the container $C_{2}$ putting two identical balls, marked with $\mathrm{L}$ and $\mathrm{R}$ respectively, inside it.

If it is chosen to move towards right, draw the numbers occurred at the positions

$$
i, i+1, i+2, \ldots \ldots \ldots \ldots . ., i+n-1
$$

in the table to obtain the $\mathrm{n}$ random three-digit numbers.

If it is chosen to move towards left, draw the numbers occurred at the positions

$$
i, i-1, i-2, \ldots \ldots \ldots \ldots . ., i-n+1
$$

in the table to obtain the $\mathrm{n}$ random three-digit numbers.

It may occur that some number or numbers among those drawn may be occurred twice. In that situation, retain only one occurrence of them and draw additional numbers appeared at the consecutive positions in the table as per requirement.

If $\mathrm{k}$ additional numbers are required to draw, then draw the numbers occurred at the positions

$$
i+n, i+n+1,, i+n+2, \ldots \ldots \ldots ., i+n+k-1
$$

if it is chosen to move towards right and draw the numbers occurred at the positions

$$
i-n, i-n-1, i-n-2, \ldots \ldots \ldots ., i-n-k+1
$$

if it is chosen to move towards left.

Drawing of random three-digit Numbers (not necessarily distinct)

In order to draw $\mathrm{n}$ random two-digit numbers which need not necessarily be distinct, from a table of random three-digit numbers, one is to proceed with the following steps $[46,56]$ :

Select one position from where to start at random by the 
similar method as in the case of drawing of distinct random twodigit numbers mentioned above. Let the ith position be selected.

Draw the number that occurs at the ith position in the table.

Choose the length of jump that is to be 1001 or more and 1999 or less at random. It can be chosen by some usual manual randomization technique of drawing one number from among the numbers

$$
101,102,103 \text {, }
$$

Let the selected length of jump be 1 .

The random selection of the length of the jump can be done by similar method as done in the selection of the starting position.

Choose whether to jump towards left or towards right. The choice can be made by the same method as in the earlier case.

If it is chosen to jump towards right, draw the numbers occurred at the positions

$$
i, i+l, i+2 l, \ldots \ldots \ldots \ldots . . ., i+(n-1) l
$$

in the table to obtain the required $\mathrm{n}$ random two-digit numbers.

If it is chosen to move towards left, draw the numbers occurred at the positions

$$
i, i-l, i-2 l, \ldots \ldots \ldots \ldots ., i-(n-1) l
$$

in the table to obtain the required $\mathrm{n}$ random three-digit numbers.

\section{Method of Drawing of Random m-Digit Numbers $(m \geq 4)$}

Now, discussion will be made on the methods of drawing of random m-digit numbers (for $m=4,5,6,7,8$, 9) using the two tables of random numbers, one of random two-digit numbers [45] and the other of random three-digit numbers [48] constructed by Chakrabarty.

\section{Drawing of random four-digit numbers}

A table of random two-digit numbers is used in drawing random four-digit numbers. Two methods have been found for drawing of random four-digit numbers [55-57].

First method of drawing: In this method, drawing of $n$ random four-digit numbers consists of the following steps [5557]:

Make a choice at random which set's two-digit numbers will be placed at the left position and which set's two-digit number will be placed at the right position while combining them in the formation of random four-digit numbers. This can be done by a binomial trial.

Draw the $1^{\text {st }}$ set of $\mathrm{n}$ random two-digit numbers from the table of random two-digit numbers by the method discussed above.

Draw the $2^{\text {nd }}$ set of $n$ random two-digit numbers from the table by the same method independently from the $1^{\text {st }}$ set.

Combine the random two-digit numbers of the $1^{\text {st }}$ set with the corresponding random two-digit numbers of the $2^{\text {nd }}$ set by the choice of the positions obtained in step (1) to obtain the $n$ random four-digit numbers.

Second method of drawing: In this method, drawing of $n$ random four-digit numbers consists of the following steps [5557]:

Draw two random two-digit numbers independently from the table of random two-digit numbers by the same method as discussed above.

Make a choice at random which set's two-digit numbers will be placed at the left position and which set's two-digit number will be placed at the right position while combining them in the formation of random four-digit numbers. This can be done by a binomial trial.

Combine the two two-digit numbers, obtained in step (1), as per the selected choice of the positions to obtain one random four-digit number.

Perform the above three steps more $(n-1)$ times to obtain more $(n-1)$ random four-digit numbers.

The random four-digit numbers obtained in step (3) \& Step (4) are the required $n$ random four-digit numbers.

Drawing of Random Five-digit Numbers: Two tables, one of random two-digit numbers and the other of random threedigit numbers, are required for drawing of random five-digit numbers. Drawing of $\mathrm{n}$ random five-digit numbers consists of the following steps $[48,56,57]$ :

Make a choice at random whether two-digit numbers will be placed at the left position or three-digit numbers will be placed at the left position while combining them in the formation of random five-digit numbers. This can be done by a binary random trial.

Draw $\mathrm{n}$ random two-digit numbers from the table of random two-digit numbers

Draw $\mathrm{n}$ random three-digit numbers from the table of random three-digit numbers.

Combine the random two-digit numbers obtained from The table of random two-digit numbers with the corresponding random three-digit numbers obtained from The table of random three-digit numbers, as per the choice of the position obtained in step (1), to obtain the $\mathrm{n}$ random five-digit numbers.

In order to draw $\mathrm{n}$ random five-digit numbers one can also proceed with the following steps: 
Draw one random two-digit numbers from the table of random two-digit numbers.

Draw one random three-digit numbers from the table of random three-digit numbers.

Make a choice at random whether two-digit number will be placed at the left position or three-digit number will be placed at the left position while combining them in the formation of random five-digit numbers. This can be done by a binary random trial.

Combine the two numbers as per the selected choice of the positions to obtain one random five-digit number.

Repeat the four steps, from the $2^{\text {nd }}$ step to the ${ }^{5 \text { th }}$ step, more $(n-1)$ times to obtain $\mathrm{n}$ random five-digit numbers.

Drawing of Random Six-digit Numbers: Random six digit numbers can be drawn either from a table of random two-digit numbers or from a table of random three-digit numbers.

Drawing from a table of random three-digit numbers: Drawing of $\mathrm{n}$ random six-digit numbers, using a table of random three-digit numbers, consists of the following steps [51,56,57]:

Make a choice at random which set's three-digit numbers will be placed at the left position and which set's three-digit number will be placed at the right position while combining them in the formation of random six-digit numbers. This can be done by a binary trial that results in two equally likely possible outcomes.

Draw the $1^{\text {st }}$ set of $\mathrm{n}$ random three-digit number from the table of random three-digit numbers.

Draw the $2^{\text {nd }}$ set of $\mathrm{n}$ random three-digit number from the same table independently of the $1^{\text {st }}$ set.

Combine the random three-digit numbers of the $1^{\text {st }}$ set with the corresponding random three-digit numbers of the $2^{\text {nd }}$ set by the choice of the positions obtained in step (1) to obtain the $n$ random six-digit numbers.

In order to draw $\mathrm{n}$ random six-digit numbers one can also proceed with the following steps:

Draw two random three-digit numbers independently from the table of random three-digit number.

Make a choice at random which three-digit number will be placed at the left position and which three-digit number will be placed at the right position while combining them in the formation of random six-digit numbers. This can be done by the binomial trial.

Combine the two three-digit numbers, obtained in step (1), as per the selected choice of the positions to obtain one random six-digit number.

Perform the above three steps more $(n-1)$ times to obtain more $(n-1)$ random six-digit numbers.
The random six-digit numbers obtained in step (3) \& Step (4) are the required $n$ random six-digit numbers.

Drawing from a table of random two-digit numbers: Drawing of $\mathrm{n}$ random six-digit numbers, using a table of random two-digit numbers, consists of the following steps $[51,56,57]$ :

Make a choice at random which set's two-digit numbers will be placed at the left position, which set's two-digit numbers will be placed at the middle position and which set's two-digit numbers will be placed at the right position while combining them in the formation of random six-digit numbers. This can be done by a random trial that results in 6 possible outcomes. Throwing of an unbiased dice, distinguishing its six sides by the six possible outcomes, can be performed in selecting the said choice.

Draw $1^{\text {st }}$ set of $\mathrm{n}$ random two-digit number from the table of random two-digit numbers.

Draw $2^{\text {nd }}$ set of $n$ random two-digit number from the table by the same steps independently of the $1^{\text {st }}$ set.

Draw $3^{\text {rd }}$ set of $\mathrm{n}$ random two-digit number from table by the same steps independently of the $1^{\text {st }}$ set $\&$ the $2^{\text {nd }}$ set.

Combine the respective random two-digit numbers of the three sets by the choice of the positions obtained in step (1) to obtain the $\mathrm{n}$ random six-digit numbers.

In order to draw $\mathrm{n}$ random six-digit numbers one can also proceed with the following steps:

Draw three random two-digit numbers from the table independently of each other.

Make a choice at random which set's two-digit numbers will be placed at the left position, which set's two-digit numbers will be placed at the middle position and which set's two-digit numbers will be placed at the right position while combining them in the formation of random six-digit numbers. This can be done by a random trial that results in six possible outcomes.

Combine the three two-digit numbers, obtained in step (1), as per the selected choice of the positions obtained in step (2), to obtain one random six-digit number.

Repeat the above three steps more $(n-1)$ times to obtain $n$ random six-digit numbers.

Drawing of Random Seven-digit Numbers: Two tables, one of random two-digit numbers and the other of random threedigit numbers, are required for drawing of random seven-digit numbers. Drawing of $\mathrm{n}$ random seven-digit numbers consists of the following steps $[53,56,58]$ :

Make a choice, from among the three sets of which one is of random three-digit numbers \& the other two are of random two-digit numbers, at random which set's numbers drawn will be placed at the left position, which set's at the middle position and which set's at the right position while combining them in the 
formation of random seven-digit numbers. This can be done by a random trial that results in six possible outcomes.

Draw the $1^{\text {st }}$ set of $\mathrm{n}$ random two-digit number from the table of random two-digit numbers.

Draw the $2^{\text {nd }}$ set of $\mathrm{n}$ random two-digit number from the table of random two-digit numbers independently of the $1^{\text {st }}$ set.

Draw a set of $\mathrm{n}$ random three-digit number from the table of random three-digit numbers.

Combine the respective numbers of the three sets by the choice of the positions obtained in step (1) to obtain the $n$ random seven-digit numbers.

In order to draw $\mathrm{n}$ random seven-digit numbers one can also proceed with the following steps:

Draw two random two-digit numbers independently from the table of random two-digit numbers.

Draw one random three-digit number from the table of random three-digit numbers.

Make a choice at random which of the three numbers drawn will be placed at the left position, which at the middle position and which at the right position while combining them in the formation of random seven-digit numbers. This can be done by a random trial that results in six possible outcomes as mentioned above.

Combine the three drawn numbers as per the selected choice of the positions to obtain one random seven-digit number.

Repeat the above steps more $(n-1)$ times to obtain $n$ random seven-digit numbers.

Drawing of Random Eight-digit Numbers: Random eightdigit numbers can be drawn a single table of random two-digit numbers or from a combination of two tables, one of random two-digit numbers and the other of random three-digit numbers $[56,58]$.

Drawing from a Table of Random Two-digit Numbers: In order to draw $n$ random eight-digit numbers from a table of random twodigit numbers one can proceed with the following steps $[56,58]$

Make a choice at random which set's two-digit numbers will be placed at the left position, which set's two-digit numbers will be placed at the $1^{\text {st }}$ middle position from the left, which set's two-digit numbers will be placed at the $2^{\text {nd }}$ middle position from the left and which set's two-digit numbers will be placed at the right position while combining them in the formation of random eight-digit numbers. This can be done by a random trial that results in 24 possible outcomes.

Draw $1^{\text {st }}$ set of $\mathrm{n}$ random two-digit number from The table of random two-digit numbers.

Draw $2^{\text {nd }}$ set of $\mathrm{n}$ random two-digit number from The table of random two-digit numbers independently of the $1^{\text {st }}$ set.
Draw $3^{\text {rd }}$ set of $n$ random two-digit number from The table of random two-digit numbers independently of the $1^{\text {st }}$ set $\&$ the $2^{\text {nd }}$ set.

Draw $4^{\text {th }}$ set of $\mathrm{n}$ random two-digit number from The table of random two-digit numbers independently of the earlier sets.

Combine the respective random two-digit numbers of the four sets by the choice of the positions obtained in step (1) to obtain the $\mathrm{n}$ random eight-digit numbers.

One can also proceed with the following steps in order to draw $n$ random eight-digit numbers from a table of random twodigit numbers [56,58]:

Draw four random two-digit numbers from the table of random two-digit numbers independently of each other.

Make a choice at random which of the four two-digit numbers will be placed at the left position, which at the $1^{\text {st }}$ middle position from the left, which at the $2^{\text {nd }}$ middle position from the left and which the right position while combining them in the formation of random eight-digit numbers. This can be done by a random trial that results in 24 possible outcomes.

Combine the four two-digit numbers, obtained in step (1), as per the selected choice of the positions obtained in step (2), to obtain one random eight-digit number.

Repeat the above three steps more $(n-1)$ times to obtain $\mathrm{n}$ random eight-digit numbers.

Drawing from a combination of one table of random twodigit numbers and one table of Three-digit Numbers: In order to draw $\mathrm{n}$ random eight-digit numbers from a combination of two tables, one of random two-digit numbers and the other of random three-digit numbers, one can proceed with the following steps $[56,58]$ :

Make a choice at random which of the three numbers drawn will be placed at the left position, which at the middle position and which at the right position while combining them in the formation of random seven-digit numbers. This can be done by a random trial that results in 6 possible outcomes.

Draw the $1^{\text {st }}$ set of $\mathrm{n}$ random three-digit number from the table of random three-digit numbers.

Draw the $2^{\text {nd }}$ set of $\mathrm{n}$ random three-digit number from the table of random three-digit numbers independently of the $1^{\text {st }}$ set.

Draw a set of $n$ random two-digit number from the table of random two-digit numbers.

Combine the respective numbers of the three sets by the choice of the positions obtained in step (1) to obtain the $n$ random eight-digit numbers.

One can also proceed with the following steps in order to draw $\mathrm{n}$ random eight-digit numbers from a combination of two tables, one of random two-digit numbers and the other of random three-digit numbers $[56,58]$ : 
Draw two random three-digit numbers independently from the table of random three-digit numbers.

Draw one random two-digit number from the table of random two-digit numbers.

Make a choice at random which of the three numbers drawn will be placed at the left position, which at the middle position and which at the right position while combining them in the formation of random eight-digit numbers. This can be done by a random trial that results in six possible outcomes as mentioned above.

Combine the three drawn numbers as per the selected choice of the positions to obtain one random eight-digit number.

Repeat the above steps more $(n-1)$ times to obtain $\mathrm{n}$ random eight-digit numbers.

Drawing of random nine-digit numbers: Random nine-digit numbers can be drawn a single table of random three-digit numbers Drawing of $\mathrm{n}$ random nine-digit numbers consists of the following steps $[56,58]$ :

Make a choice at random which set's will be placed at the left position, which set's two-digit numbers will be placed at the middle position and which set's two-digit numbers will be placed at the right position while combining them in the formation of random six-digit numbers. This can be done by a random trial which results in 6 possible outcomes namely.

Draw $1^{\text {st }}$ set of $n$ random three-digit number from the table of random three-digit numbers.

Draw $2^{\text {nd }}$ set of $n$ random three-digit number from the table of random three-digit numbers independently of the $1^{\text {st }}$ set.

Draw $3^{\text {rd }}$ set of $n$ random three-digit number from the table of random three-digit numbers independently of the $1^{\text {st }}$ set \& the $2^{\text {nd }}$ set.

Combine the respective random three-digit numbers of the three sets by the choice of the positions obtained in step (1) to obtain the $\mathrm{n}$ random nine-digit numbers.

One can also proceed with the following steps in order to draw $\mathrm{n}$ random nine-digit numbers from a table of table of random three-digit numbers steps [56-58]:

Draw 3 random three-digit numbers from the table independently of each other.

Make a choice at random which three-digit numbers will be placed at the left position, which at the middle position and which at the right position while combining them in the formation of random nine-digit numbers. This can be done by a random trial that results in 6 possible outcomes.

Combine the 3 three-digit numbers, obtained in step (1), as per the selected choice of the positions obtained in step (2), to obtain one random nine-digit number.
Repeat the above three steps more $(n-1)$ times to obtain $n$ random nine-digit numbers.

\section{Conclusion}

In this paper, steps of works/computations involved in the respective methods of drawing of random $\mathrm{m}$-digit numbers (for $m=2,3,4,5,6,7,8,9$ ), from a single table of random twodigit numbers and/or from a single table of random threedigit numbers and/or from a combination of the two which one is suitable, have been outlined/described only. Numerical applications of the methods are available in the respective articles [45-48,51-56,58]. Methods of drawing of random $\mathrm{m}$-digit numbers for $\mathrm{m}>9$ can be derived in a similar manner. The significance of this paper is that the methods developed here enables us to draw random m-digit numbers (for $2<\mathrm{m}<9$ ) with the help of either a single table of random two-digit numbers or from a single table of random three-digit numbers or from a combination of the two depending upon $\mathrm{m}$.

\section{References}

1. Tippett LHC (1927) random sampling numbers. tracts for computers no. 15, Cambridge University Press, Cambridge, England, UK.

2. Fisher RA, Yates F (1938) Statistical tables for biological agricultural and medical research. ( $6^{\text {th }}$ edn), Longman Group Limited, England, UK. Pp. 134 - 139.

3. Hald A (1952) Table of random numbers, In: Hald A (Ed.), Statistical Tables and Formulas, Wiley, New Jersey, USA.

4. Hill ID, Hill PA (1977) Tables of Random Times. UK.

5. Kendall MG, Smith BB (1938) Randomness and random sampling numbers. J Royal Statistical Society 101(1): 147-166.

6. Kendall MG, Smith BB (1939) A table of random sampling numbers: tracts for computers no. 24. Cambridge University Press, Cambridge, England,UK.

7. Mahalanobis PC (1934) Tables of random samples from a normal population. Sankya 1(2/3): 289-328.

8. Manfred Mohr (1971) Le petit livre de nombres au hasar. Édition d'artiste, Paris, UK.

9. Moses EL, Oakford VR (1963) Tables of Random Permutations. George allen \& unwin, Crows Nest, Australia.

10. Quenouille MH (1959) Tables of random observations from standard distributions. Biometrika, 46(1/2): 178-202.

11. Rand Corporation (1955) A Million Random Digits. Free Press, Glenoe, Ireland.

12. Rao CR, Mitra SK, Matthai A (1966) Random Numbers and Permutations. Statistical Publishing Society, Calcutta, India.

13. Rohlf FJ, Sokal RR (1969) Ten thousand random digits, In: Rohlf, Sokal, (Eds.), Statistical Tables, Freeman, USA.

14. Royo J, Ferrer S (1954) Tables of random numbers obtained from numbers in the spanish national lottery. Trabajos de Estadistica 5: 247-256.

15. Snedecor GW, Cochran WG (1967) Statistical Methods. Iowa State University Press, Ames, Iowa, USA.

16. Sarmah BK, Chakrabarty D (2014) Testing of randomness of the number generated by fisher and yates. IJESRT 3(11): 632-636. 
17. Sarmah BK, Chakrabarty D (2014) Examination of proper randomness of the number generated by LHC Tippett. IJESRT 3(12): 661-668.

18. Sarmah BK, Chakrabarty D (2015) Testing of proper randomness of the table of number generated by MG Kendall and B Babington Smith (1939). IJESRT 4(2): 260-282.

19. Sarmah BK, Chakrabarty D, Barman N (2015) Testing of proper randomness of the table of number generated by rand corporation (1955). IJESM 5(1): 97-119.

20. Sarmah BK, Chakrabarty D (2015) Testing of randomness of the numbers generated by fisher and yates. Arya Bhatta J Math Info 7(1) 87-90.

21. Sarmah BK, Chakrabarty D (2015) Examination of proper randomness of the numbers generated by LHC Tippett (1927). IOSR Journal of Mathematics 11(3): 35-37.

22. Sarmah BK, Chakrabarty D (2015) Examination of proper randomness of the numbers generated by rand corporation (1955) random number table: t-Test. IJIRSET 4(10): 9536-9540.

23. Sarmah BK, Chakrabarty D (2015) Testing of proper randomness of the numbers generated by kendall and babington Smith: t-Test. Arya Bhatta J Math Info 7(2): 365-368.

24. Sarmah BK, Chakrabarty D (2015) Examination of proper randomness of numbers of MG Kendall and B Babington Smith's random numbers table: Run test. IJMRME 1(2): 223-225.

25. Sarmah BK, Chakrabarty D (2015) Testing of randomness of the numbers generated by fisher and yates: Run test. IJIR SET 4(12): 11956-11958

26. Chakrabarty D (2010) Chakrabarty's definition of probability: prope randomness of fisher and yates random number table. Int J Agricult Stat Sci 6(2): 461-469.

27. Chakrabarty D, Sarmah BK (2017) Comparison of degree of randomness of the tables of random numbers due to tippet, fisher \& yates, kendall \& smith and rand corporation. JRSS 10(1): 27-42.

28. Chakrabarty D (2017) Deviation test: comparison of degree of randomness of the tables of random numbers due to tippet, fisher \& yates, kendall \& smith and rand corporation. SM Journal of Biometrics \& Biostatistics 2(3): 1014.

29. Chakrabarty D (2018) Random numbers tables due to tippet, fisher \& yates, kendall \& smith and rand corporation: comparison of degree of randomness by run test. Journal of Biostatistics and Biometric Applications 3(1): 1-7.

30. Chakrabarty D (2004) A theoretical definition of probability based on common sense. Bulletin of Pure and Applied Sciences - E 23E(2): 343-349.

31. Chakrabarty D (2005) Probability: link between the classical definition and the empirical definition. J Ass Sc Soc 13-18.

32. Chakrabarty D (2006) Non-equally likely outcomes: the classical definition of probability. Bulletin of Pure and Applied Sciences - E 25E(2): 471-481.

33. Chakrabarty D (2007) Empirical definition of probability: special case of its theoretical definition. Int J Agricult Stat Sci, 3(1): 261-267.

34. Chakrabarty D (2008) Bernoulli's definition of probability: special case of its chakrabarty's definition. Int J Agricult Stat Sci 4(1): 23-27.

35. Chakrabarty D (2009) Probability: Chakrabarty's definition from its classical definition. Int J Agricult Stat Sci 5(1): 181-187.

36. Chakrabarty D (2010) A method of determining the value of probability. Int J for Statiscians 1(1): 5-7.
37. Chakrabarty D (2010) Probability as the maximum occurrence of relative frequency. Arya Bhatta J Math Info 2 (2): 339- 344.

38. Chakrabarty D (2010) Chakrabarty's definition of probability: additive and multiplicative laws. Bulletin of Pure and Applied Sciences-E 29E(2): 265-274.

39. Chakrabarty D (2011) Probability in ideal situation and in practical situation. Arya Bhatta J Math Info 3(1): 161-168.

40. Sarmah BK, Chakrabarty D (2016) A table of two-digit random numbers. Arya Bhatta J Math Info 8(1): 63-74.

41. Sarmah BK, Chakrabarty D (2016) Construction of a table of threedigit random numbers. IJCR 8(3): 28425-28431.

42. Sarmah BK, Chakrabarty D (2017) Manual method of construction: table of random three-digit numbers. Arya Bhatta Journal of Mathematics \& Informatics 9(1): 135-148.

43. Chakrabarty D (2013) One table of two-digit random numbers. Arya Bhatta J Math Info 5(1): 141-152.

44. Chakrabarty D (2013) One table of three-digit random numbers. Arya Bhatta J Math Info 5(2): 285-294.

45. Chakrabarty D (2016) One more table of random two-digit numbers IJARSET 3(3): 1667-1678.

46. Chakrabarty D (2016) One more table of random three-digit numbers IJARSET 3(4): 1851-1869.

47. Chakrabarty D, Sarmah BK (2017) Manual method of construction table of random two-digit numbers. Biometrics \& Biostatistics International Journal 5(5): 00144.

48. Chakrabarty D (2016) Drawing of random five-digit numbers from tables of random two-digit and three-digit numbers IJARSET 3(7): 2385-2306.

49. Chakrabarty D (2016) Drawing of random six-digit numbers from tables of random three-digit numbers. IJARSET 3(8): 2507-2515.

50. Chakrabarty D (2016) Drawing of random six-digit numbers from tables of random two-digit numbers. IJARSET 3(9): 2643-2655.

51. Chakrabarty D (2016) Drawing of random six-digit numbers from a single table of random two-digit numbers. IJARSET 3(10): 27432753.

52. Chakrabarty D (2016) Drawing of random six-digit numbers from a single table of random three-digit numbers. IJARSET 3(11): 29052914.

53. Chakrabarty D (2016) Drawing of random seven-digit numbers from tables of random two-digit numbers and of three-digit numbers. IJARSET 3(12): 3003-3012.

54. Chakrabarty D (2016) drawing of random four-digit numbers from independent tables of random two-digit numbers in selection of random sample. Biometrics \& Biostatistics International Journal 4(7): 00118

55. Chakrabarty D (2017) Drawing of random four-digit numbers from a single table of random two-digit numbers. IJARSET 4(2): 3377-3387.

56. Chakrabarty D (2017) Selection of Random Sample: Drawing of Random Numbers (ISBN: 978-3659959806) Lambart Academic Publishing, Germany, UK.

57. Chakrabarty D (2017) Drawing of random nine-digit numbers from a single table of random three-digit numbers. IJARSET 4(3): 35663575.

58. Dhritikesh C (2017) Recent developments on drawing of multi-digit random numbers from single table of random two-digit numbers and of random three-digit numbers. BBOAJ 2(5): 1-16. 
Your next submission with Juniper Publishers will reach you the below assets

- Quality Editorial service

- Swift Peer Review

- Reprints availability

- E-prints Service

- Manuscript Podcast for convenient understanding

- Global attainment for your research

- Manuscript accessibility in different formats ( Pdf, E-pub, Full Text, Audio)

- Unceasing customer service

Track the below URL for one-step submission https://juniperpublishers.com/online-submission.php 